\title{
Role of Computed Tomography in the Assessment of the Orbital Space Occupying Lesions
}

\author{
Mohammed Ismail ${ }^{1}$, Priyanka B ${ }^{2}$, M R Shashikumar ${ }^{3}$, N L Rajendrakumar ${ }^{4}$, C P Nanjaraj ${ }^{5}$

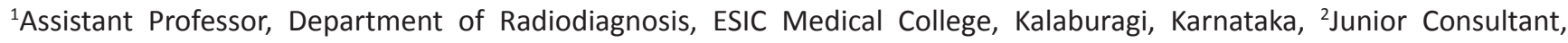 \\ Department of Radiodiagnosis, Gleneagles Global Hospital, Chennai, Tamil Nadu, ${ }^{3}$ Professor, Department of Radiodiagnosis, \\ JSS Medical College, Mysuru, Karnataka, ${ }^{4}$ Professor, Department of Radiodiagnosis, Mysore Medical College and Research \\ Institute, Mysuru, Karnataka, ${ }^{5}$ Professor, Department of Radiodiagnosis, Mysore Medical College and Research Institute, \\ Mysuru, Karnataka, India
}

Corresponding author: Dr Priyanka B, \#10, Chitra Avenue, New Perungalathur, Chennai - 63, Tamil Nadu

DOI: http://dx.doi.org/10.21276/ijcmsr.2018.3.4.21

How to cite this article: Mohammed Ismail, Priyanka B, M R Shashikumar, N L Rajendrakumar, C P Nanjaraj. Role of computed tomography in the assessment of the orbital space occupying lesions. International Journal of Contemporary Medicine Surgery and Radiology. 2018;3(4):D90-D94.

\section{A B S T R A C T}

Introduction: A broad spectrum of orbital lesions produces symptoms like proptosis, diplopia and visual defects. CT helps to disclose the enigma posed by these symptoms. Information regarding the anatomical location of orbital space occupying lesions, involvement of various intra orbital structures and extension of pathology into peri-orbital regions could be routinely obtained.

Material and methods: Patients with clinically suspected orbital space occupying lesion were included in the study and CT examination was tailored accordingly. The images were evaluated to characterize the distribution, morphology, involvement of adjacent structures and associated findings of the orbital space occupying lesions.

Results: Our study included a total of 50 cases, commonest age group was 1 to 20 years. Proptosis and Swelling were the major presenting complaints. Unilateral masses were noted in $92 \%$ and bilateral in $8 \%$. Extraconal orbital lesions were found in $60 \%$ of cases and intraconal lesions in $22 \%$ of cases, $10 \%$ of cases involved both. Primary orbital lesions were seen in $74 \%$ of cases, secondary orbital lesions were seen in $18 \%$ of cases and metastases were seen in $8 \%$ of cases. Vasculogenic lesions were the most common lesions accounting for $20 \%$, followed by secondary tumours (18\%), lymphoreticular lesions (14\%) and cystic lesions (12\%). Lacrimal gland fossa lesions accounted for $8 \%$ of cases.

Conclusion: CT is useful in characterization and preoperative evaluation of the orbital space occupying lesions. The main advantages of CT are availability and shorter imaging time; thus, it has become an established investigation in the work-up of orbital space occupying lesions.

Key words: Computed Tomography, Orbit, Proptosis, Lymphoma, Metastases, Histopathology.

\section{INTRODUCTION}

The orbit is a pyramidal shaped recess that contains and protects the globe, extraocular muscles, blood vessels, nerves, adipose and connective tissues, and most of the lacrimal apparatus. Diseases can affect the bone or any of the soft tissue structures. ${ }^{1}$

The orbit is the site of wide variety of diseases, majority of which represent primary conditions, but a significant number of orbital diseases arise within the surrounding bones or compartments or may be associated with systemic diseases. Orbital space occupying lesions comprise a broad spectrum of benign and malignant entities. ${ }^{2}$ Orbital lesions produce few symptoms like proptosis, diplopia and visual defects. Cross-sectional imaging plays a pivotal role in characterization of these lesions and in evaluation of disease extent, supplementing clinical examination. ${ }^{2}$ Computed tomography helps to disclose the enigma posed by these symptoms and accurately discern the number, size, nature and extent of the orbital space occupying lesions.

The anatomical relation of the orbit to paranasal sinuses makes it susceptible to pathologies of the adjacent sinuses. CT provides better delineation of bony orbit and the adjoining soft tissues. ${ }^{1} \mathrm{CT}$ can produce better images because of natural contrast provided by retrobulbar fat, bone and air in the adjacent paranasal sinuses. CT provides a detailed cross-sectional anatomy of the entire globe and an excellent topographic visualization.

While both CT and MRI provides cross sectional depictions, the main advantages of CT are availability and shorter imaging time, besides being less expensive. ${ }^{3} \mathrm{CT}$ has become an established investigation in the work-up of orbital space occupying lesions. Information regarding the anatomical location of orbital lesions, involvement of various intra orbital structures and extension of pathology into periorbital regions such as sinuses and intracranial compartment could be routinely obtained. CT is helpful in the preoperative evaluation of the orbital space occupying lesion, thus aiding 
the surgical approach.

Hence this study was undertaken to assess and characterize the orbital space occupying lesions based on the computed tomography features and correlate with clinical and histopathological findings.

\section{MATERIAL AND METHODS}

This prospective study was conducted at Department of Radio-Diagnosis, Mysore Medical College and Research Institute, Mysore for the duration of one year from January 2015 to December 2015.

Total 50 patients were included in the study after approval from institutional ethics committee and patient consent was obtained. Any mass originating in the orbit or invading the orbit from paranasal sinuses, nose or brain were included in the study. And patients with orbital trauma/inflammatory conditions were excluded.

A detailed history and clinical examination were carried out. All the cases were evaluated using GE High Speed Dual-Slice CT Scanner. Diagnosis were confirmed either by imaging features of the lesions or fine needle aspiration cytology / histopathological reports wherever feasible.

After positioning of the patient, a lateral scanogram was taken. Thin axial sections parallel to the orbitomeatal line were obtained. Coronal sections were taken, whenever required. Study was performed before and after administration of intravenous contrast. Low osmolar non-ionic contrast medium was used. CT scan images were viewed in head window, soft tissue window and bone window.

\section{STATISTICAL ANALYSIS}

Descriptive statistical methods were applied in the present study. All the statistical calculations were done through SPSS for windows (v 20.0)

\section{RESULTS}

A total of 50 patients with orbital space occupying lesions were evaluated using computed tomography. Age at presentation ranged from 7 months to 75 years. Maximum numbers of patients were seen in the age group of 1 to 20 years accounting for $32 \%$. Orbital space occupying lesions were more common in males, constituting 26 (52\%) cases and females constituted 24 (48\%) cases. Figure 1 depicts

\begin{tabular}{|l|c|c|}
\hline Orbital masses & Number & Percentage \\
\hline Cystic lesions & 6 & $12 \%$ \\
\hline Vasculogenic lesions & 10 & $20 \%$ \\
\hline Peripheral nerve sheath lesions & 3 & $6 \%$ \\
\hline Optic nerve and meningeal lesions & 3 & $6 \%$ \\
\hline Lymphoreticular Lesions & 7 & $14 \%$ \\
\hline Lacrimal gland (fossa) lesions & 4 & $8 \%$ \\
\hline Osseous and fibro-osseous lesions & 1 & $2 \%$ \\
\hline Lipocytic and myxoid tumors & 1 & $2 \%$ \\
\hline Metastatic tumors to the orbit & 4 & $8 \%$ \\
\hline $\begin{array}{l}\text { Secondary orbital lesions from } \\
\text { adjacent structures }\end{array}$ & 9 & $18 \%$ \\
\hline Others & 2 & $4 \%$ \\
\hline \multicolumn{2}{|c|}{ Table-1: Spectrum of Orbital space occupying lesions. } \\
\hline
\end{tabular}

clinical presentation of orbital space occupying lesions. Unilateral orbital lesions were seen in 46 (92\%) cases, of which, involvement of the right side was seen in 27 (54\%)

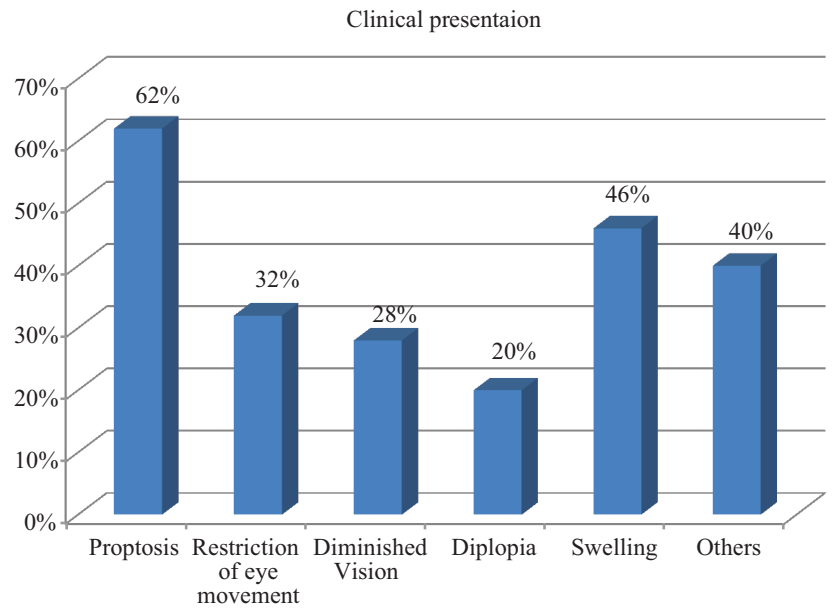

Figure-1: Clinical presentation of orbital space occupying lesions.

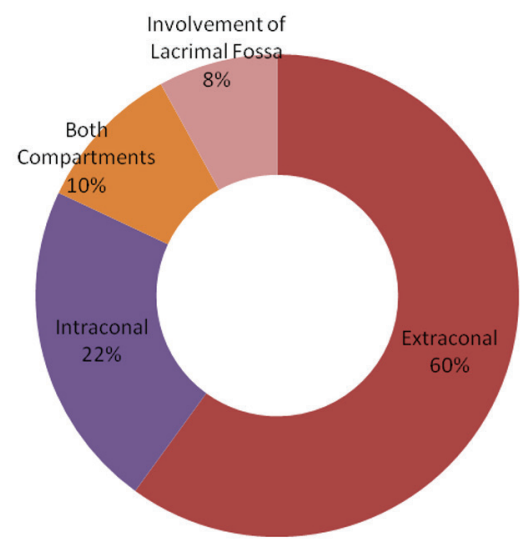

Figure-2: Location of SOL in orbit

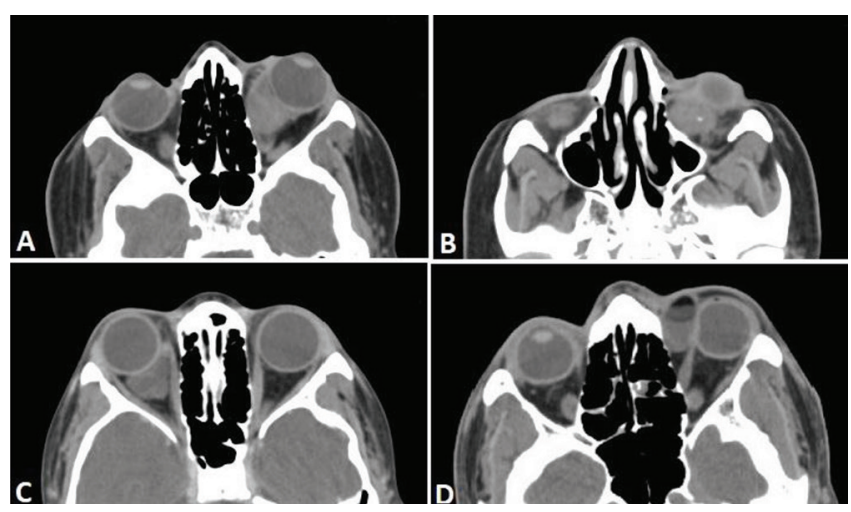

Figure-3: Cavernous hemangioma - Axial CT sections ( $A$ and $B$ ) in soft tissue windows, showing a well-defined hypodense lesion in intraconal space. A tiny calcification is seen (B). Lymphangioma - Axial CT sections (C) in soft tissue window, showing a lobulated infiltrating intraconal cystic lesion.

Axial CT sections (D) in soft tissue window, showing well defined rounded extraconal orbital SOL with internal fat density consistent with dermoid cyst. Scalloping of adjacent orbital walls was noted. 


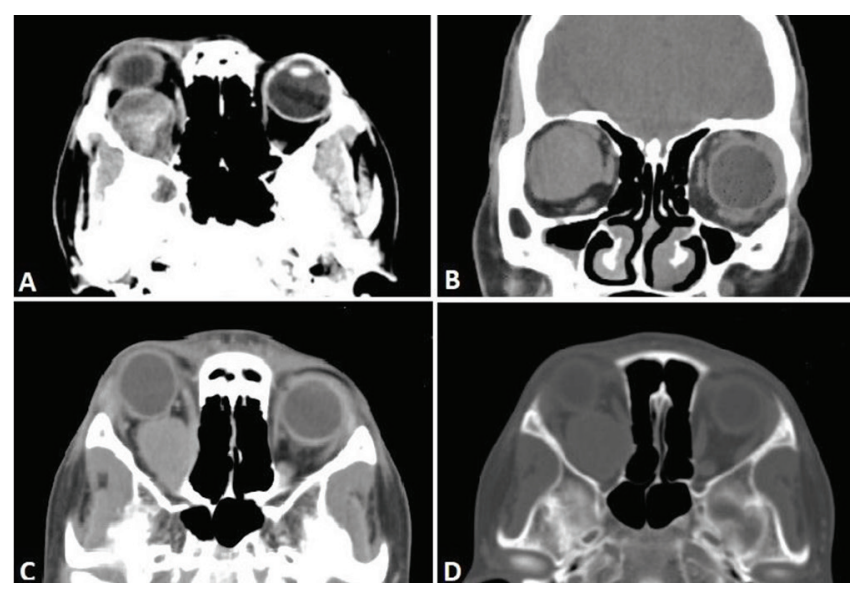

Figure-4: Schwannoma - Axial(A)and Coronal CT sections (B) show a well circumscribed heterogeneously enhancing mass lesion in intraconal compartment of the right orbit. Optic nerve meningioma - Axial(C and D) CT sections demonstrate a well defined oval homogenously enhancing lesion in the intraconal region of the right orbit along the optic nerve extending upto the optic foramen with expansion of the bony orbit and optic foramen

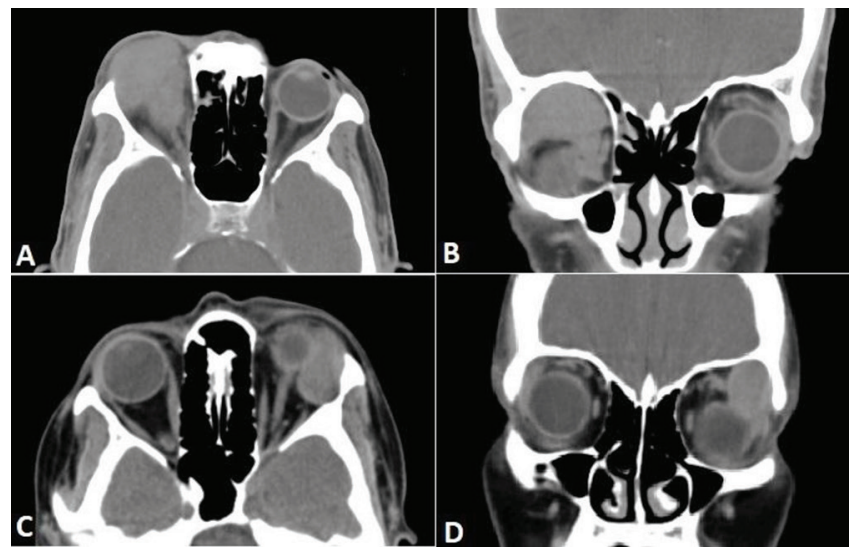

Figure-5: Lymphoma - Axial and coronal CT sections (A and $\mathrm{B}$ ) showing an ill-defined homogenously isodense(A) lesion in the extraconal compartment of the right orbit with involvement of superior rectus.

Lacrimal gland pleomorphic adenoma - A 45 yr old female patient presented with swelling and dryness of the left eye, Axial and Coronal CT (C and D) sections show a well circumscribed isodense lesion at the lacrimal fossa of the left orbit with remodelling of the superolateral wall of the orbit.

cases and left side in 19(38\%) cases and bilateral involvement was noted in $4(8 \%)$ cases. Figure 2 depicts location of SOL in orbit.

On NECT, isodense lesions were common, noted in 32\% of cases and hypodense were noted in $18 \%$ of cases, of which 4 $(8 \%)$ cases were of fat density. Calcifications were found in 10(20\%) cases. On CECT, homogenous enhancement was seen in $44 \%$ of cases, heterogenous enhancement was seen in $40 \%$, peripheral rim enhancement was seen in $12 \%$ cases. No enhancement was noted in $2 \%$ of lesions.

Extraocular muscle involvement was seen in 16(32\%) cases in the present study, predominantly in malignant lesions such as lymphoma, metastasis and propagated secondary lesions.

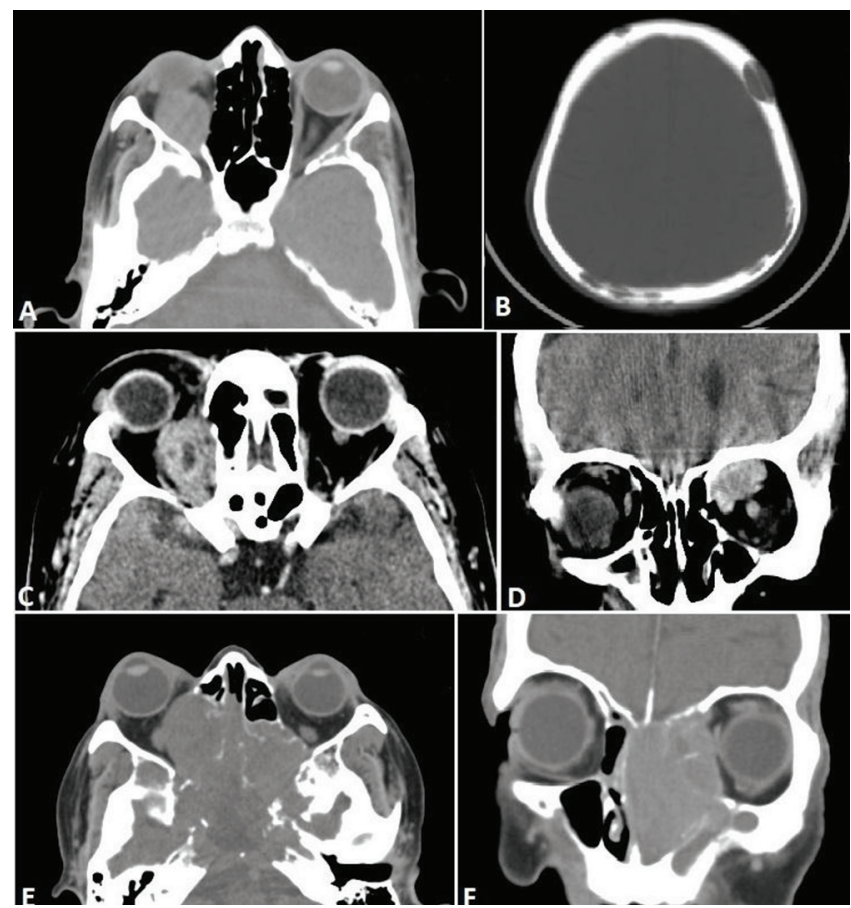

Figure-6: Metastases -In a known case of Carcinoma Breast, Axial CT(A) section shows an intraconal lesion on the right side with bone window(B) showing lytic lesions in the calvarium. In a known case of Carcinoma Prostate, Axial(C) and coronal(D) CT sections show an ill-defined heterogenous lesion in the extraconal compartment of the right orbit with involvement of the superior and medial recti muscles.

Secondary orbital lesions - Axial CT section(E) shows large nasopharyngeal mass with bone destruction and orbital extension on right side. Coronal CT(F) section shows heterogenous sinonasal mass extending into the orbit with bone erosion.

Erosion/destruction of bones was seen in 9(18\%) cases which was mainly caused by malignant lesions and remodelling/ scalloping of underlying bones was seen in $11(22 \%)$ cases.

Primary orbital lesions were seen in 37 (74\%) cases, secondary orbital lesions were seen in $9(18 \%)$ cases and metastases were seen in $4(8 \%)$ cases. Fifty six percent ( 5 cases) of the secondary orbital lesions were sinonasal and 22\% (2 cases) were nasopharyngeal and intracranial each. Table 1 depicts spectrum of orbital space occupying lesions.

\section{DISCUSSION}

Many comprehensive studies have investigated the cause of proptosis and incidence of orbital space occupying lesions. The present study was undertaken with the objectives of localization of orbital space occupying lesions, study their morphological features and compare the CT diagnosis with histopathological findings wherever possible.

We examined 50 patients of orbital space occupying lesions irrespective of age. Age at presentation ranged from 7 months to 75 years. Maximum numbers of patients were seen in the age group of 1 to 20 years accounting for $32 \%$. Out of 50 patients, 26(52\%) were males and 24 (48\%) were females.

In this study we found proptosis to be the most common 
clinical finding, seen in 31(62\%) patients, while swelling was the second most common finding seen in 23(46\%) patients, followed by restriction of movements and diminished vision (Figure 1). This correlates with Amit Gupta et $\mathrm{al}^{4}$ who found proptosis as the most common clinical finding. There were 46 (92\%) unilateral cases and 4(8\%) bilateral cases, comparable to study by Demirci $\mathrm{H}$ et $\mathrm{al} .^{5}$

Vasculogenic lesions were found in $20 \%$ of the cases, capillary hemangioma was found in 2 (4\%) cases. Findings are comparable to Mafee et al. ${ }^{6}$ Cavernous hemangioma was found to be the commonest benign lesion in adult age group, noted in $6(12 \%)$ cases, however Shields et $\mathrm{al}^{7}$ in their survey reported cavernous hemangioma in $6 \%$ of cases. (Figure $3 \mathrm{~A}$ and $3 \mathrm{~B}$ )

Lymphangioma was seen in $2(4 \%)$ cases, extraconal in one and intraconal in the other, both showed minimal enhancement on contrast study (Figure 3C). Amit Gupta et $\mathrm{al}^{4}$ found lymphangioma in $11.5 \%$ cases. Murat Tunc et $\mathrm{al}^{8}$ studied 26 cases of lymphangioma. Most of the lesions (73\%) were involving both compartments and the remaining lesions isolated to either extraconal or intraconal space.

Dermoid cyst was found in $5(10 \%)$ cases, it was the most common benign orbital tumor in pediatric age group, which is comparable to Amit Gupta et $\mathrm{al}^{4}$ and Shields et al. ${ }^{7}$ All the lesions were extraconal and hypodense on NECT (Figure 3D).

In the present study, peripheral nerve sheath lesions were found in $3(6 \%)$ cases, neurofibroma in $2(4 \%)$ cases and schwannoma was seen in $1(2 \%)$ case (Figure $4 \mathrm{~A}$ and $4 \mathrm{~B}$ ). The age of presentation was third and fourth decades. Both neurofibromas were extraconal and schwannoma was intraconal. One case of neurofibroma was seen in a known case of neurofibromatosis I. Findings were similar to Shields et $\mathrm{al}^{7}$ who reported peripheral nerve lesions in $2 \%$ cases.

Optic nerve glioma was found in $1(2 \%)$ case and presented in the second decade of life, it was similar to a study conducted by Amit Gupta et $\mathrm{al}^{4}$ in which optic nerve gliomas accounted for $2.8 \%$ of cases. However Shields et $\mathrm{al}^{7}$ reported optic nerve glioma in $4 \%$ of cases. Optic nerve meningioma was found in 2 (4\%) cases (Figure 4C and 4D). The patients were females, presented in 2 nd and 3rd decade with proptosis and diminution of vision. Contrast CT scan showed moderately enhancing eccentric masses in both the cases. Findings were similar to Shields et $\mathrm{al}^{7}$ and Mafee et al. ${ }^{6}$

Orbital lymphoma accounted for 7 (14\%) cases in our study (Figure $5 \mathrm{~A}$ and $5 \mathrm{~B}$ ). It was the most common lesion in older population. Age of presentation was 4th to 6th decade. Findings were comparable to Shields et $\mathrm{al}^{7}$ and Demirci $\mathrm{H}$ et al..$^{5}$ Most of the lesions were extraconal, isodense / hyperdense and showed enhancement on contrast study.

In the present study, lacrimal gland tumors accounted for 4 (8\%) cases. Among these tumors, pleomorphic adenoma (Figure 5C and 5D) accounted for 2(4\%) cases, lymphoma and adenoid cystic carcinoma accounted for 1 (2\%) case each. Above findings were comparable to studies by Shields et $\mathrm{al}^{7}$ and Eagle et al. ${ }^{9}$

In the present study one (2\%) case of retinoblastoma was noted causing destruction of the optic globe and extending into the orbit with involvement of extraocular muscles and optic nerve. Amit Gupta et $\mathrm{al}^{4}$ reported retinoblastoma (4.2\%) as the most common ocular tumor infiltrating into the orbit. Colobomatous cyst and rhabdomyosarcoma constituted 1 (2\%) case each. Fibrous dysplasia was found in 1 (2\%) case, presented in third decade of life. Similar findings were seen in the study conducted by Vashisht et al. ${ }^{10}$ Angiomyxoma orbit was noted in 1 (2\%) case. The lesion was intraconal and heterogenous in appearance with heterogenous enhancement. Bajaj et $\mathrm{al}^{11}$ in their study reported angiomyxoma as a rare, locally aggressive orbital tumor, occurring in the third to fourth decade of life.

Metastases were found in 4 (8\%) cases out of which metastases from carcinoma of breast accounted for $2(4 \%)$ cases and metastasis from lung and prostate carcinoma accounted for one case each (Figure 6A - 6D). Findings were comparable to Shields et $\mathrm{al}^{7}$ and Valenzuela AA et al. ${ }^{12}$

Secondary orbital lesions were found in 9 (18\%) cases. Maxillary carcinoma (6\%), nasopharyngeal carcinoma (4\%), ethmoidal carcinoma (2\%), ethmoidal mucocele (2\%), esthesioneuroblastoma (2\%) and intracranial meningioma (2\%) were various entities in our study (Figure 6E and 6F). Findings were comparable to study by Rauniyar RK et al. ${ }^{13}$

In the present study, primary orbital lesions were seen in $37(74 \%)$ cases, secondary orbital tumors were seen in $9(18 \%)$ cases and metastases in $4(8 \%)$ cases. Benign lesions accounted for 28(56\%) cases and malignant tumors for 22 (44\%) cases. Malignant lesions were more common in elderly and lymphoma was the most common malignancy. Our study correlated with studies by Shields et $\mathrm{al}^{7}$ and Rodahl E et al. ${ }^{14}$ Out of total 50 cases, 40 cases were verified histologically. In the rest of the cases further evaluation was done using ultrasound and MRI, final diagnosis was reached. In five cases final diagnosis was different from the CT diagnosis. Thus, CT showed an overall diagnostic accuracy of $90 \%$. Computed tomography was accurate in delineation of the nature of lesion and extension in most of the cases. The present study correlated well with the study of Moseley and Bull ${ }^{15}$, they found CT to be positive in $87.5 \%$ of their histopathologically verified orbital tumors. In the study conducted by Sabarwal KK et $\mathrm{al}^{16} \mathrm{CT}$ diagnosis of orbital tumors correlated well with histopathological evaluation in $78.26 \%$ of cases.

\section{CONCLUSION}

CT and MRI are the preferred modalities in the evaluation of orbital space occupying lesions. CT characterizes the lesion and facilitates in arriving at a diagnosis. It is helpful in the preoperative evaluation of the orbital space occupying lesion, thus aiding in surgical approach. The main advantages of CT are availability and shorter imaging time, besides being less expensive, thus it has become an established investigation in the work-up of orbital space occupying lesions.

\section{REFERENCES}

1. Mafee MF, Som PM. Embryology, Anatomy, and Imaging of the Eye and Orbit. In: Som PM, Cutin HD (eds), Head and neck Imaging. $5^{\text {th }}$ ed. St. Louis: Mosby; 2011.

2. Tina DT, Divakar G, Roberta WD, Dirk K, Yoshimi A. 
Orbital Neoplasms in Adults: Clinical, Radiologic, and Pathologic Review. RadioGraphics 2010; 30(1):461-82.

3. Zeynel A Karcioglu. Orbital tumors, Diagnosis and treatment. Springer; 2005.

4. Amit Gupta, Sima Das, Rameshmurthy, Geeta KV, Santosh GH. Orbital tumors in children. AIOC; 2010. pp.491-3.

5. Demirci H, Shields CL, Shields JA, Honavar SG, Mercado GJ, Tovilla JC. Orbital tumors in the older adult population. Ophthalmology 2002;109(2):243-8.

6. Mafee MF, Putterman A, Valvassori GE. Orbital spaceoccupying lesions: role of computed tomography and magnetic resonance imaging. An analysis of 145 cases. Radiol Clin North Am 1987;25(3):529-59.

7. Shields JA, Shields CL, Scartozzi R. Survey of 1264 patients with orbital tumors and simulating lesions: the 2002 Montgomery Lecture, part 1. Ophthalmology 2004;111(5):997-1008.

8. Tunç M, Sadri E, Char DH. Orbital lymphangioma: an analysis of 26 patients. British Journal of Ophthalmology 1999;83(1):76-80.

9. Shields CL, Shields JA, Eagle RC, Rathmell JP. Clinicopathologic review of 142 cases of lacrimal gland lesions. Ophthalmology 1989;96(5):431-5.

10. Vashisht S, Goulati RK, Dayal Y, Bhargava S. Impact of computerised axial tomography on orbital diagnosis. Indian J Ophthal 1983; 31(4):347-52.

11. Bajaj MS, Mehta M, Kashyap S, Pushker N, Lohia $\mathrm{P}$, Chawla B et al., Clinical and pathologic profile of angiomyxomas of the orbit. Ophthal Plast Reconstr Surg 2011;27(2):76-80.

12. Valenzuela AA, Archibald CW, Fleming B, Ong L, O'Donnell B, Crompton JJ, et al. Orbital metastasis: clinical features, management and outcome. Orbit 2009;28(2-3):153-9.

13. Rauniyar RK, Thakur SD, Panda A, Sharma U. Radiological Evaluation of Orbit in cases of Proptosis in Eastern Nepal. NJR 2011;1(1):8-14.

14. Rodahl EL, Bertelsen T, Seland J, Arnes JB, Mork S. Orbital tumors. Tidsskr Nor Laegeforen 2000 Nov 30;120(29):3518-23.

15. Moseley IF, Bull JWD. Computerised axial tomography, carotid angiography and orbital phlebography in the diagnosis of space occupying lesions of the orbit. In: Salmon G (ed), Advances in cerebral angiography. New York: Springer; 1975. p.361.

16. Sabharwal KK, Chouhan AL, Jain S. CT Evaluation of proptosis IJRI 2006; 16(3):4683-8.

Source of Support: Nil; Conflict of Interest: None

Submitted: 23-10-2018; Accepted: 25-11-2018; Published online: 07-12-2018 